\title{
STOCHASTIC GROWTH: ASYMPTOTIC DISTRIBUTIONS
}

\author{
JOHN STACHURSKI
}

\begin{abstract}
This note studies conditions under which sequences of capital per head generated by stochastic optimal accumulation models have law of large numbers and central limit properties. The regularity condition used on the productivity shock is somewhat different to that of previous studies. In particular, no restrictions are placed on its support. Instead, an "average contraction" property is required on the law of motion. Journal of Economic Literature Classification Numbers: C51, C62, O41.
\end{abstract}

\section{INTRODUCTION}

A discrete-time stochastic growth model is characterized by a sequence of probability distributions $\left(\varphi_{t}\right)_{t \geq 0}$ on the state space, one for each point in time. The entire sequence of these distributions can be calculated ex ante from the hypothesized laws of motion, the distribution of the disturbance term, and a given initial value $x_{0}$ for the state. A trajectory or time series for the model is a sequence $\left(x_{t}\right)_{t \geq 0}$ on the state space generated by a simultaneous draw from each of these distributions $\left(x_{t}\right.$ is drawn according to $\varphi_{t}$ ).

For Markovian models, calculation of the sequence of distributions $\left(\varphi_{t}\right)$ can be performed iteratively. In other words, $\varphi_{t+1}$ can be calculated from knowledge of $\varphi_{t}$ alone, rather than the entire history of the sequence prior to $t$. Equivalently, there exists an operator mapping the

Key words and phrases. Stochastic growth, law of large numbers, central limit theorem.

The author would like to thank John Creedy for helpful comments. 
collection of all distributions on the state space into itself with the property that the image of the current distribution $\varphi_{t}$ is the next period distribution $\varphi_{t+1}$. An equilibrium for the growth model, if it exists, is a distribution $\bar{\varphi}$ that is invariant under this operation. The equilibrium is globally stable if $\varphi_{t} \rightarrow \bar{\varphi}$ in some appropriate topology, regardless of initial conditions.

Conditions for existence, uniqueness and stability of such equilibria have been studied by many authors. ${ }^{1}$ If convergence to a limiting distribution does occur, it is reasonable to ask whether sample averages of time series generated by the model converge to the mean of the limiting distribution, and whether average deviations from this population mean are eventually normally distributed. In other words, do law of large numbers (LLN) and central limit theorem (CLT) results hold for the stochastic process generated by the model?

If an LLN condition holds, then it is possible to test a given theoretical model by comparing the mean of the limiting distribution with a sample average from a sufficiently large data set generated by the system under study. Conversely, suppose that an expression is available for the mean of the hypothetical model in terms of its parameters. Then the implied equality of this expression and the sample mean calculated from data provides another equation for identifying parameter values. If, in addition, a CLT result is available, then inference can be drawn as to the likelihood of various parameter values.

In the existing literature, a well-known LLN condition for correlated processes on compact state space is given in Stokey et al. [14, Theorem 14.7]. In the context of stochastic growth, compactness of the state space implies that the support of the productivity shock is bounded.

\footnotetext{
${ }^{1}$ The seminal papers are Brock and Mirman [4], Mirman [8], [9] and Mirman and Zilcha [10]. For more recent results and an introduction to the literature, see, e.g., Stachurski [12].
} 
Bhattacharya and Majumdar [2, Theorem 2.2] provide a "splitting condition" under which CLT results hold for systems on a bounded interval. Ergodicity in moments for the Solow-Swan model with a shock that is unbounded above but cannot be arbitrarily small is investigated in Binder and Pesaran [3]. Evstigneev and Flåm [5] and Amir and Evstigneev [1] give CLT results for the asymptotic distributions of aggregate rewards accumulated along equilibrium and optimal paths in a stochastic Polterovich setting.

In this note, results recently obtained by Łoskot and Rudnicki [7, Theorem 1, Theorem 3] are applied to provide sufficient conditions under which stochastic growth models have LLN and CLT properties. ${ }^{2}$ The application involves translating the models into a space isometrically isomorphic to the real line, where the conditions of Łoskot and Rudnicki are all satisfied. No restrictions are required on the support of the disturbance term.

Section 2 formulates the problem for an abstract dynamic economy, and states the conditions developed by Łoskot and Rudnicki. Section 3 applies this framework to growth systems and examines two cases that satisfy the conditions.

\section{The ModeL}

In what follows, $(\Omega, \mathcal{F}, \mathbf{P})$ is a probability space, $(X, d)$ is a metric space and $\mathcal{B}(X)$ denotes the Borel subsets of $X$.

2.1. Perturbed dynamic economies. Consider a dynamic economic model evolving on $X$. Preferences, technology, market conditions and other primitives of the model imply a transition rule $T$, and that the

\footnotetext{
${ }^{2}$ Previously, the techniques of Loskot and Rudnicki have been applied to computation of entropy for iterated function systems (Slomczynski, Kwapien and Zyczkowski [11]) and analysis of neurodynamics (Feng and Brown [6]).
} 
action of $T$ on the state is perturbed at each transition by an $X$-valued shock $\varepsilon_{t}$. That is,

$$
T: X \times X \ni\left(x_{t}, \varepsilon_{t}\right) \mapsto x_{t+1} \in X .
$$

The uncorrelated sequence of shocks $\left(\varepsilon_{t}\right)$ is drawn according to $\mathbf{P}$. More precisely, $\varepsilon: \Omega \ni \omega \mapsto \varepsilon(\omega) \in X$ is an $\mathcal{F}$-measurable function, and the time $t$ value $\varepsilon\left(\omega_{t}\right) \in X$ is realized by an independent draw from $\Omega$ by $\mathbf{P}$. As anticipated by the above notation, the realized value $\varepsilon\left(\omega_{t}\right)$ is often written simply as $\varepsilon_{t}$.

Analogous to the definition in Brock and Mirman [4, p. 492], an equilibrium for the economy $(1)$ is a probability measure $\bar{\varphi}: \mathcal{B}(X) \rightarrow[0,1]$ such that

$$
\bar{\varphi}(B)=\int_{X}\left[\int_{\Omega} \mathbf{1}_{B}[T(x, \varepsilon(\omega))] \mathbf{P}(d \omega)\right] \bar{\varphi}(d x), \quad \forall B \in \mathcal{B}(X) .
$$

The intuition is as follows. Suppose that the current distribution for the state variable $x$ is $\bar{\varphi}$. The inner integral gives the probability of traveling from current state $x$ into Borel set $B$, and the outer integral sums across all $x \in X$, weighted by the probability $\bar{\varphi}(d x)$ of $x$ occurring as the current state. Thus the right hand side is the probability of the state being in $B$ next period, given that the current distribution is $\bar{\varphi}$. The left hand side is the probability of being in $B$ this period. If (2) holds for all $B \in \mathcal{B}(X)$, then the probabilistic laws that govern the current state do not change from period to period.

2.2. Asymptotic distributions. A real function $g$ on $X$ is called a Lipshitz function on $X$ if there exists a constant $K$ such that $\mid g(x)-$ $g\left(x^{\prime}\right) \mid \leq K d\left(x, x^{\prime}\right)$ for any $x, x^{\prime} \in X$. The map $T$ in (1) is called an average contraction on $X$ if there exists a measurable function $\lambda: X \rightarrow$ $\mathbb{R}$ such that $E(\lambda)=\int_{\Omega} \lambda[\varepsilon(\omega)] \mathbf{P}(d \omega)<1$ and

$$
d\left(T(x, z), T\left(x^{\prime}, z\right)\right) \leq \lambda(z) d\left(x, x^{\prime}\right) \quad \forall x, x^{\prime}, z \in X
$$

It is shown in Eoskot and Rudnicki [7, Theorem 1] that if $X$ is complete and separable, $T$ is an average contraction on $X$, an initial point $x_{0} \in X$ 
is given, and there exists at least one point $\bar{x} \in X$ such that

$$
\int_{\Omega} d(\bar{x}, T[\bar{x}, \varepsilon(\omega)]) \mathbf{P}(d \omega)<\infty,
$$

then a unique equilibrium distribution $\bar{\varphi}$ exists. Moreover, the sequence of state variables $\left(x_{t}\right)$ satisfies

$$
\frac{1}{N} \sum_{t=0}^{N-1} g\left(x_{t}\right) \rightarrow \int_{X} g(x) \bar{\varphi}(d x) \text { a.s., } \quad N \rightarrow \infty,
$$

for any Lipschitz function $g: X \rightarrow \mathbb{R}$.

If, in addition, $E\left(\lambda^{2}\right)<1$ and

$$
\int_{\Omega}[d(\bar{x}, T(\bar{x}, \varepsilon(\omega)))]^{2} \mathbf{P}(d \omega)<\infty
$$

also holds, then the sequence $(1 / \sqrt{N}) \sum_{t=0}^{N-1} g\left(x_{t}\right)$ converges in distribution to a normal random variable with mean $\int_{X} g(x) \bar{\varphi}(d x)$ and variance $\sigma^{2} \geq 0$ (Eoskot and Rudnicki [7, Theorem 3]).

\section{Applications}

The average contraction condition stated above is rather strong. For stochastic growth models, the condition fails in the standard Euclidean metric whenever Inada restrictions hold. However, there exists a complete separable metric space in which a number of models satisfy the condition. This proves sufficient to obtain LLN and CLT results.

Let the state space $X$ be the positive real numbers. Consider the onesector optimal growth problem

$$
\begin{aligned}
& \max \mathrm{E}\left[\sum_{t=0}^{\infty} \beta^{t} u\left(c_{t}\right)\right] \\
& \text { s.t. } k_{t+1}=f\left(k_{t}, \varepsilon_{t}\right)-c_{t}
\end{aligned}
$$

where $c_{t} \in X$ is consumption, $k_{t} \in X$ is capital per head, $\beta \in(0,1)$ is a discount factor, and $f: X \times X \rightarrow X$ and $u: X \rightarrow \mathbb{R}$ are the production and utility functions respectively. ${ }^{3}$ The utility function $u$ is assumed to

\footnotetext{
${ }^{3}$ For background on the optimal growth problem see, e.g., Stokey et al. [14].
} 
satisfy $u^{\prime}(0)=\infty$, which assures interiority of solutions, and therefore eliminates the possibility of zero savings or consumption. The shocks $\varepsilon_{t} \in X$ are independent draws from $\mathbf{P}$ as before.

The solution to the planning problem, if it exists, is an optimal policy $g: X \rightarrow X$, which associates realized output $f\left(k_{t}, \varepsilon_{t}\right)$ with optimal current consumption $c_{t}$. Optimal consumption $g\left(f\left(k_{t}, \varepsilon_{t}\right)\right)$ can then be substituted into (6) to obtain the closed loop law of motion for the system, which is in the form of (1).

Using this framework, the techniques of Łoskot and Rudnicki are now applied to establish LLN and CLT results for two well-known parameterizations.

Example 3.1. Consider first the unit-elastic model, where $u(c)=$ $\log c, f(k, \varepsilon)=A k^{\alpha} \varepsilon, \alpha \in(0,1)$. The productivity parameter $A$ is strictly positive. For such a specification, the optimal policy consumes a fraction $1-\alpha \beta$ of realized output $A k^{\alpha} \varepsilon$, implying the law of motion

$$
k_{t+1}=\alpha \beta A k_{t}^{\alpha} \varepsilon_{t}
$$

Define a binary $\rho$ on $X \times X$ by $\rho(x, y)=|\log (x / y)|$. Evidently $\rho$ is a distance on $X$ in the metric sense. Moreover, the space $(X, \rho)$ is isometrically isomorphic to $\mathbb{R}$ under the mapping $x \mapsto \log x$ when the latter space is endowed with its usual Euclidean metric. Hence $(X, \rho)$ is a complete, separable metric space.

It is straightforward to show that (7) is an average contraction on $X$ for the metric $\rho$, with $\lambda(z) \equiv \alpha$. If $E|\log \varepsilon|$ is finite, then condition (3) holds for $\bar{x}=1$, implying the existence of a unique equilibrium $\bar{\varphi}: \mathcal{B}(X) \rightarrow[0,1]$, and the LLN result

$$
\frac{1}{N} \sum_{n=0}^{N-1} g\left(k_{n}\right) \rightarrow \int_{0}^{\infty} g(k) \bar{\varphi}(d k) \text { a.s. }
$$


for the process $\left(k_{t}\right)$, where $k_{0} \in X$ is any strictly positive initial condition and $g$ is any Lipschitz function on $X{ }^{4}$

Evidently $E\left(\lambda^{2}\right)<1$ also holds. If, in addition, $E\left[(\log \varepsilon)^{2}\right]$ is also finite, then (4) is satisfied, and $(1 / \sqrt{N}) \sum_{t=0}^{N-1} g\left(k_{t}\right)$ is asymptotically normal with mean $\int_{0}^{\infty} g(k) \bar{\varphi}(d k)$.

Remark 3.1. The conditions $E|\log \varepsilon|<\infty$ and $E\left[(\log \varepsilon)^{2}\right]<\infty$ can be viewed as restrictions on the left- and right-hand tails of the distribution. See the discussion in Stachurski [13, Remark 4.1].

Example 3.2. The second example is from Mirman and Zilcha [10, Example A, p. 333]. The state space $X$, the shock $\varepsilon$, the discount factor $\beta$, the productivity parameter $A$ and utility $u(c)=\log c$ are as before. Let $\alpha$ be a Borel function from $X$ into $(0,1)$. The production function is $(k, \varepsilon) \mapsto A k^{\alpha(\varepsilon)}$. For such a specification, the law of motion is

$$
k_{t+1}=\bar{\alpha} \beta A k_{t}^{\alpha\left(\varepsilon_{t}\right)}, \quad \bar{\alpha}=\int_{\Omega} \alpha[\varepsilon(\omega)] \mathbf{P}(d \omega) .
$$

Once again, the system is an average contraction on $X$ under the metric $\rho$, this time using $\lambda(z)=\alpha(z)$. (Evidently $E(\lambda)=\bar{\alpha}<1$ ). Note that in this case (3) holds for any shock $\varepsilon$ when $\bar{x}=1$. Hence for any strictly positive initial condition $k_{0}$, a unique equilibrium distribution $\bar{\varphi}$ exists and (8) holds for arbitrary Lipschitz function $g$.

Moreover, $E\left(\lambda^{2}\right)<1$, and (4) holds for any shock $\varepsilon$ when $\bar{x}=1$, implying that $(1 / \sqrt{N}) \sum_{t=0}^{N-1} g\left(k_{t}\right)$ is asymptotically normal with mean $\int_{0}^{\infty} g(x) \bar{\varphi}(d x)$.

\section{REFERENCES}

[1] R. Amir and I. V. Evstigneev, A functional central limit theorem for equilibrium paths of economic dynamics, J. Math. Econ., 33 (2000), 81-99.

[2] R. Bhattacharya and M. Majumdar, On a class of stable random dynamical systems: Theory and applications, J. Econ. Theory, 96 (2001), 208-229.

\footnotetext{
${ }^{4}$ The equilibrium is in fact globally stable in total variation norm. See Stachurski [13, Proposition 4.1].
} 
[3] M. Binder and M. H. Pesaran, Stochastic growth models and their econometric implications, J. Econ. Growth 4 (2) (1999), 139-183.

[4] W. A. Brock and L. Mirman, Optimal economic growth and uncertainty: The discounted case, J. Econ. Theory 4 (1972), 479-513.

[5] I. V. Evstigneev and S. D. Flåm, The turnpike property and the central limit theorem in stochastic models of economic dynamics, in "Statistics and Control of Stochastic Processes" (Yu M. Kabanov, B. L. Rozovskii and A. N. Shiryaev, Eds.), p. 63-101, World Scientific, London, 1997.

[6] J. F. Feng and D. Brown, Fixed-point attractor analysis for a class of neurodynamcis, Neural Computation 10 (1998), 189-213.

[7] K. Loskot and R. Rudnicki, Limit Theorems for Stochastically Perturbed Dynamical Systems, J. Appl. Probab. 32 (1995), 459-469.

[8] L. J. Mirman, On the existence of steady state measures for one sector growth models with uncertain technology, Int. Econ. Rev. 13 (1972), 271-286.

[9] L. J. Mirman, The steady state behavior of a class of one sector growth models with uncertain technology, J. Econ. Theory 6 (1973), 219-242.

[10] L. J. Mirman and I. Zilcha, On optimal growth under uncertainty, J. Econ. Theory 11 (1975), 329-339.

[11] W. Slomczynski, J. Kwapien and K. Zyczkowski, Entropy computing via integration over fractal measures, Chaos 10 (2000), 180-188.

[12] J. Stachurski, Stochastic optimal growth with unbounded shock, J. Econ. Theory, Forthcoming 2001.

[13] J. Stachurski, Log-linearization of perturbed dynamical systems, with applications to optimal growth, University of Melbourne Department of Economics Research Paper No. 788, May 2001.

[14] N. L. Stokey, R. E. Lucas and E. C. Prescott, "Recursive Methods in Economic Dynamics," Harvard University Press, Massachusetts, 1989.

Department of Economics, The University of Melbourne, ViC 3010

E-mail address: j.stachurski@pgrad.unimelb.edu.au 